\title{
Non-Commutative Vector Bundles for Non-Unital Algebras
}

\author{
Adam RENNIE and Aidan SIMS
}

School of Mathematics and Applied Statistics, University of Wollongong, Northfields Ave 2522, Australia

E-mail: renniea@uow.edu.au,asims@uow.edu.au

Received December 13, 2016, in final form June 12, 2017; Published online June 16, 2017 https://doi.org/10.3842/SIGMA.2017.041

\begin{abstract}
We revisit the characterisation of modules over non-unital $C^{*}$-algebras analogous to modules of sections of vector bundles. A fullness condition on the associated multiplier module characterises a class of modules which closely mirror the commutative case. We also investigate the multiplier-module construction in the context of bi-Hilbertian bimodules, particularly those of finite numerical index and finite Watatani index.
\end{abstract}

Key words: Hilbert module; vector bundle; multiplier module; Watatani index

2010 Mathematics Subject Classification: 57R22; 46L85

\section{Introduction}

The Serre-Swan theorem says that the Hilbert modules over unital commutative $C^{*}$-algebras that can be realised as the modules of sections of locally trivial vector bundles over compact spaces are precisely the finite projective modules. By direct analogy, we define non-commutative vector bundles over unital $C^{*}$-algebras to be finite projective modules, this definition being justified by the connection to the non-commutative definition of $K$-theory.

This note revisits the question of the correct notion of a non-commutative vector bundle over a non-unital $C^{*}$-algebra. We prove the equivalence of several conditions on a Hilbert module $E$ over a non-unital $C^{*}$-algebra $A$ that, when $A$ is commutative, characterise modules of sections of vector bundles. This extends previous partial characterisations [9]. In particular, our results apply to suspensions of finitely generated projective modules over unital $C^{*}$-algebras. This is an important motivation since it allows us to apply techniques like those of [10] to study CuntzPimsner algebras of suspended $C^{*}$-correspondences, and thereby to bootstrap computational techniques from even $K$-groups and Kasparov groups to their odd counterparts.

We then investigate the structure of multiplier modules associated to bimodules that are biHilbertian in the sense of [6]. We prove that, under mild hypotheses, the bi-Hilbertian structure and finite numerical index pass to the multiplier module. We also establish that, by contrast, the multiplier module frequently does not have finite Watatani index. Indeed the multiplier module has finite Watatani index if and only if it is finitely generated and projective as a right-Hilbert module over the multiplier algebra, which in turn holds if and only if it is full as a left-Hilbert module over the multiplier algebra.

We start by briefly recalling what is already known. Then we prove our first result, Theorem 3.1, which shows that if $E$ is a Hilbert module over a $\sigma$-unital $C^{*}$-algebra $A$, then its multiplier module is full as a left-Hilbert $\operatorname{End}_{A}(E)$-module if and only if $E$ is a finitely generated projective module over a suitable unitisation of $A$. We discuss some consequences of this result. In particular, by applying our results to the setting of commutative $C^{*}$-algebras, we prove that every locally trivial vector bundle $V$ over a locally compact space $X$ of finite topological 
dimension extends to a locally trivial vector bundle $V^{c}$ over some compactification $X^{c}$ of $X$. The compactification $X^{c}$ required, and the isomorphism class of the extension $V^{c}$, depend on a choice of frame for $V$, which we illustrate by example.

We then recall the notion of a bi-Hilbertian bimodule [6]. We prove that if $E$ is countably generated with injective left action and finite Watatani index, then the bi-Hilbertian structure, and also finite numerical index, pass from $E$ to its multiplier module in a natural way. We then describe a number of conditions that are equivalent to the multiplier module being finitely generated and projective, and show how this applies to modules over commutative $C^{*}$-algebras.

\section{Finite projective modules and non-unital analogues}

Throughout the paper, $A$ denotes a $\sigma$-unital $C^{*}$-algebra. Given a right $C^{*}$ - $A$-module $E$, we denote the $C^{*}$-algebra of adjointable operators on $E$ by $\operatorname{End}_{A}(E)$. For $e, f \in E$, the rank-1 endomorphism $g \mapsto e \cdot(f \mid g)_{A}$ is denoted by $\Theta_{e, f}$, and is adjointable with adjoint $\Theta_{f, e}$. We write $\operatorname{End}_{A}^{0}(E)$ for $\overline{\operatorname{span}}\left\{\Theta_{e, f}: e, f \in E\right\}$, the closed *-ideal of compact endomorphisms in $\operatorname{End}_{A}(E)$. We write $\ell^{2}(A)$ for the standard $C^{*}$-module over $A$; that is $\ell^{2}(A)$ is equal to $\left\{\xi: \mathbb{N} \rightarrow A \mid \sum_{n=1}^{\infty} \xi_{i}^{*} \xi_{i}\right.$ converges in $\left.A\right\}$ with inner product $(\xi \mid \eta)_{A}=\sum_{i} \xi_{i}^{*} \eta_{i}$. We say that a (right) inner product module $E$ over a $C^{*}$-algebra $A$ is full if the closed span of the inner products $(e \mid f)_{A}$ is all of $A$.

In keeping with the preceding paragraph, throughout the paper, by a vector bundle over a locally compact Hausdorff space $X$, we will mean a locally trivial finite-rank complex vector bundle equipped with a continuous family of inner products. If $X$ is paracompact, then every finite rank vector bundle over $X$ admits such a family of inner products.

A frame for a right $C^{*}$-A-module $E$ is a sequence $\left\{e_{j}\right\}_{j \geq 1} \subset E$ such that

$$
\sum_{j \geq 1} \Theta_{e_{j}, e_{j}} \text { converges strictly to } \operatorname{Id}_{E} \text {. }
$$

If $\left\{e_{j}\right\}_{j \geq 1}$ is a frame for $E$, then $E$ is generated as a right $A$-module by the $e_{j}$, so it is countably generated.

Any frame $\left\{e_{j}\right\}$ for $E$ determines a stabilisation map in the sense of Kasparov: there is an adjointable map $v: E \rightarrow \ell^{2}(A)$ such that

$$
v(e)=\left(\left(e_{j} \mid e\right)_{A}\right)_{j \geq 1} \quad \text { for all } e \in E .
$$

We have $v^{*} v=\operatorname{Id}_{E}$, and so $p:=v v^{*}$ is a projection in $\operatorname{End}_{A}\left(\ell^{2}(A)\right)$; specifically, $p$ satisfies

$$
(p \xi)_{i}=\sum_{j}\left(e_{i} \mid e_{j}\right)_{A} \xi_{j} \quad \text { for all } \xi \in \ell^{2}(A) .
$$

One of the fundamental points of contact between non-commutative geometry and classical geometry is the celebrated Serre-Swan theorem. Given a vector bundle $V \rightarrow X$ over a compact space $X$, we write $\Gamma(X, V)$ for the space of continuous sections of $V$.

Theorem 2.1 ([11]). Let $X$ be a compact Hausdorff space. A (right) $C(X)$-module $M$ is finitely generated and projective if and only if there is a vector bundle $V \rightarrow X$ such that $M \cong \Gamma(X, V)$.

We can remove the word "projective" from the statement of Theorem 2.1 if we instead consider full inner-product modules over $C(X)$ - or more generally inner-product modules $E$ such that $(E \mid E)_{C(X)}$ is a unital, and hence complemented, ideal of $C(X)$. This is because every full finitely generated right inner product $C(X)$-module can be made into a $C^{*}$-module $M$ which, by Kasparov's stabilisation theorem, is automatically projective. 
When $X$ is non-compact, the natural $C_{0}(X)$-module arising from a vector bundle $V \rightarrow X$ is the module $\Gamma_{0}(X, V)$ of continuous sections of $V$ such that $x \mapsto(\xi(x) \mid \xi(x))$ belongs to $C_{0}(X)$. Important examples of this are restrictions $V \rightarrow X$ of vector bundles $V^{c} \rightarrow X^{c}$ over some compactification $X^{c}$ of $X$. The following non-unital Serre-Swan theorem characterises the algebraic structure of such modules.

Theorem 2.2 ([9, Theorem 8]). Let $X$ be a locally compact Hausdorff space and $X^{c}$ a compactification of $X$. Set $A=C_{0}(X)$ and $A_{b}=C\left(X^{c}\right)$. A right $A$-module $E$ is of the form $p A^{n}$ for some projection $p \in M_{n}\left(A_{b}\right)$ if and only if there is a (locally trivial) vector bundle $V \rightarrow X^{c}$ such that $E \cong \Gamma_{0}\left(X,\left.V\right|_{X}\right)$.

Theorem 2.2 provides a reasonable algebraic characterisation of modules of the form $\Gamma_{0}(X, V)$ where the vector bundle $V$ extends to a bundle over some compactification of $X$. We use this to motivate our definition of the non-commutative analogue.

Recall that a unitisation of a nonunital $C^{*}$-algebra $A$ is an embedding $\iota: A \hookrightarrow A_{b}$ of $A$ as an essential ideal of a unital $C^{*}$-algebra $A_{b}$.

Definition 2.3. Let $A$ be a nonunital separable $C^{*}$-algebra and $\iota: A \hookrightarrow A_{b}$ a unitisation of $A$. An $A_{b}$-finite projective $A$-module is a right $A$-module that is isomorphic to $p A^{n}$ for some $n \in \mathbb{N}$ and some projection $p \in M_{n}\left(A_{b}\right)$.

To state our main result, we need to recall how to embed a Hilbert $A$-module $E$ in the associated multiplier module, a tool used frequently in the Gabor-analysis literature (see, for instance, $[1,7])$.

Definition 2.4. Given a right $C^{*}$-A-module $E$, recall that the linking algebra $\mathcal{L}(E)$ is the algebra

$$
\mathcal{L}(E)=\operatorname{End}_{A}^{0}(E \oplus A)=\left\{\left(\begin{array}{cc}
T & e \\
\bar{f} & a
\end{array}\right): T \in \operatorname{End}_{A}^{0}(E), a \in A, e \in E, \bar{f} \in \bar{E}\right\},
$$

where $\bar{E}$ is the conjugate module (a left $A$-module). Let $r=\operatorname{Id}_{E} \oplus 0 \in \operatorname{Mult}(\mathcal{L}(E))$ and $s=0 \oplus 1_{\operatorname{Mult}(A)} \in \operatorname{Mult}(\mathcal{L}(E))$ and define

$$
\operatorname{Mult}(E)=r \operatorname{Mult}(\mathcal{L}(E)) s .
$$

Then $\operatorname{Mult}(E)$ is a right $C^{*}$-Mult $(A)$-module with right action implemented by right multiplication in $\operatorname{Mult}(\mathcal{L}(E))$, and right inner-product $(e \mid f)_{\operatorname{Mult}(A)}=e^{*} f$.

Writing $\operatorname{Hom}_{A}(A, E)$ for the Banach space of adjointable operators from $A$ to $E$, we then have $\operatorname{Mult}(E) \cong \operatorname{Hom}_{A}(A, E)$ : for any $m \in \operatorname{Mult}(E)$, the map $a \mapsto m a$ belongs to $\operatorname{Hom}_{A}(A, E)$, and conversely if $m \in \operatorname{Hom}_{A}(A, E)$, then there is a multiplier of $\mathcal{L}(E)$ such that

$$
m\left(\begin{array}{ll}
T & e \\
\bar{f} & a
\end{array}\right)=\left(\begin{array}{cc}
m \circ(f \mid \cdot)_{A} & m a \\
0 & 0
\end{array}\right) .
$$

For $e, f \in \operatorname{Mult}(E)$, let $\Theta_{e, f}$ be the associated rank-one operator on $\operatorname{Mult}(E)$. We show that $E \subseteq \operatorname{Mult}(E)$ is invariant for $\Theta_{e, f}$. To see this, fix $g \in E$, use Cohen factorisation to write $g=g^{\prime} \cdot a$ for some $g^{\prime} \in E$ and $a \in A$. Regarding $g^{\prime}$ as an element of $\operatorname{Mult}(E)$, we calculate

$$
\Theta_{e, f}(g)=e \cdot\left(f \mid g^{\prime} \cdot a\right)=e \cdot(f \mid g) a \in \operatorname{Mult}(E) \cdot A,
$$

which is equal to $E$ by [1, Remark $3.2(\mathrm{a})]$.

We deduce that $\left.\Theta_{e, f}\right|_{E}$ is adjointable with adjoint $\left.\Theta_{f, e}\right|_{E}$. Hence $\left.\Theta_{e, f}\right|_{E} \in \operatorname{End}_{A}(E)$. Abusing notation a little, we will just write $\Theta_{e, f}$ for this operator on $E$ henceforth. The left inner product 
$\operatorname{End}_{\text {Mult }(A)}^{0}(\operatorname{Mult}(E))(e \mid f)=\Theta_{e, f}$ gives a norm on $\operatorname{Mult}(E)$ equivalent to that coming from the right $\operatorname{Mult}(A)$-valued inner product. This can be seen directly from the linking algebra picture above.

The inclusion $\operatorname{End}_{\text {Mult }(A)}^{0}(\operatorname{Mult}(E)) \hookrightarrow \operatorname{End}_{A}(E)$ is injective, and so we can regard Mult $(E)$ as a left $\operatorname{End}_{A}(E)-C^{*}$-module. We will denote the left inner product by $\operatorname{End}_{A}(E)(\cdot \mid \cdot)$; we then have $\operatorname{End}_{A}(E)(e \mid f)=\left.\Theta_{e, f}\right|_{E}$ for $e, f \in \operatorname{Mult}(E)$.

\section{$3 \quad$ Finite projectivity and the multiplier module}

Theorem 3.1. Let $A$ be a non-unital $\sigma$-unital $C^{*}$-algebra and let $E$ be a right $C^{*}$-A-module. The following are equivalent.

1. There is a finite subset $F \subseteq \operatorname{Mult}(A)$ such that, putting $A_{b}:=C^{*}(F \cup A) \subseteq \operatorname{Mult}(A)$, the module $E$ is $A_{b}$-finite projective.

2. The module $E$ is $\operatorname{Mult}(A)$-finite projective.

3. The module $\operatorname{Mult}(E)$ is finitely generated and projective as a right $\operatorname{Mult}(A)$ module.

4. The module $\operatorname{Mult}(E)$ is full as a left $C^{*}$-module over $\operatorname{End}_{A}(E)$.

Proof. The universal property of $\operatorname{Mult}(A)$ shows that for any unitisation $A_{b}$ of $A$ as in (1), we have an inclusion $A_{b} \hookrightarrow \operatorname{Mult}(A)$. Thus $p A_{b}^{n} \otimes_{A_{b}} \operatorname{Mult}(A) \cong p \operatorname{Mult}(A)^{n}$ and so (1) implies (2).

For (2) implies (1), we observe that since $p \in M_{n}(\operatorname{Mult}(A))$, it has finitely many matrix entries $\left(p_{i, j}\right)_{i=1}^{n}$. Let $F=\left\{p_{i, j}: i, j \leq n\right\} \cup\left\{1_{\operatorname{Mult}(A)}\right\}$, and let $A_{b}=C^{*}(A \cup F) \subseteq \operatorname{Mult}(A)$. Then $A_{b}$ is a unitisation of $A, p \in M_{n}\left(A_{b}\right)$, and $E \cong p A^{n}$ as a right $A$-module as required.

For (2) implies (3), recall that we may identify $\operatorname{Mult}(E)$ with $\operatorname{Hom}_{A}(A, E)$, from which we deduce that $\operatorname{Mult}\left(p A^{n}\right) \cong p \operatorname{Mult}(A)^{n}$.

To see that (3) implies (2), fix a finite frame $\left\{\xi_{j}\right\}$ for $\operatorname{Mult}(E)$ as a right-Hilbert $\operatorname{Mult}(A)$ module. By Kasparov's stabilisation theorem applied to this frame, there exist an integer $n \geq 1$, a projection $p \in M_{n}(\operatorname{Mult}(A))$, and an isomorphism $\rho: \operatorname{Mult}(E) \rightarrow p \operatorname{Mult}(A)^{n}$ of $\operatorname{right} \operatorname{Mult}(A)$ modules. We claim that $\rho(E)=p A^{n}$.

To see this, first fix $e \in E$. Apply the strong form [8, Proposition 2.31] of Cohen factorisation to write $e=e^{\prime} \cdot\left(e^{\prime} \mid e^{\prime}\right)$ for some $e^{\prime} \in E$. Then $\rho(e)=p \rho(e)=p \rho\left(e^{\prime}\right) \cdot\left(e^{\prime} \mid e^{\prime}\right)$. Since $\left(e^{\prime} \mid e^{\prime}\right) \in A$ and since $\operatorname{Mult}(E) \cdot A=E$, we deduce that $\rho(e) \in p A^{n}$. So $\rho(E) \subseteq p A^{n}$. For the reverse inclusion, we fix $a \in p A^{n}$, and use Cohen factorisation in $p A^{n}$ to write $a=a^{\prime} \cdot a^{\prime \prime}$ for some $a^{\prime} \in p A^{n}$ and $a^{\prime \prime} \in A$. Write $a^{\prime}=\rho(\xi)$ for some $\xi \in \operatorname{Mult}(E)$. Using again that $\operatorname{Mult}(E) \cdot A \subseteq E$, we see that $a=\rho(\xi) \cdot a^{\prime \prime}=\rho\left(\xi \cdot a^{\prime \prime}\right) \in \rho(E)$.

To see that (3) implies (4), suppose that $\operatorname{Mult}(E)$ is finitely generated and projective as a right $\operatorname{Mult}(A)$-module. Then $\operatorname{End}_{\operatorname{Mult}(A)}(\operatorname{Mult}(E))=\operatorname{End}_{\operatorname{Mult}(A)}^{0}(\operatorname{Mult}(E))$, and so $\operatorname{End}_{A}(E) \subseteq$ $\operatorname{End}_{\operatorname{Mult}(A)}(\operatorname{Mult}(E))$ belongs to the range of the outer product. So Mult $(E)$ is full as a left $C^{*}$-module over $\operatorname{End}_{A}(E)$.

Finally, to see that (4) implies (3), note that associativity of multiplication in $\operatorname{Mult}(\mathcal{L}(E))$ shows that

$$
\xi \cdot(\eta \mid \zeta)_{\operatorname{Mult}(A)}=\operatorname{End}_{A}(E)(\xi \mid \eta) \cdot \zeta \quad \text { for all } \xi, \eta, \zeta \in \operatorname{Mult}(E)
$$

So for $\xi, \eta \in \operatorname{Mult}(E)$, the action of $\operatorname{End}_{A}(E)(\xi \mid \eta)$ is implemented by the generalised compact operator $\Theta_{\xi, \eta} \in \operatorname{End}_{\operatorname{Mult}(A)}^{0}(\operatorname{Mult}(E))$. Since $\operatorname{Mult}(E)$ is full as a left $\operatorname{End}_{A}(E)$-module, the identity operator $\operatorname{Id}_{E}$ is in the range of $\operatorname{End}_{A}(E)(\cdot \mid \cdot)$, and is therefore a compact operator on Mult $(E)$. Since $\operatorname{Id}_{E} \cdot e=e=\operatorname{Id}_{\text {Mult }(E)} e$ for all $e \in \operatorname{Mult}(E)$, we deduce that $\operatorname{Id}_{\operatorname{Mult}(E)}$ is a compact operator. So [2, Theorem 8.1.27] implies that $\operatorname{Mult}(E)$ is finitely generated. 
Remark 3.2. Suppose that $A$ and $B$ are $C^{*}$-algebras and $E_{A}$ and $E_{B}$ are right-Hilbert modules over $A$ and $B$ respectively that satisfy the equivalent conditions of Theorem 3.1. Then the external tensor product $(E \otimes F)_{A \otimes B}$ satisfies the same conditions. For if $E \cong p A^{n}$ for some projection $p \in M_{n}(\operatorname{Mult}(A))$ and $F \cong q B^{m}$ with $q \in M_{m}(B)$, then $E \otimes F \cong(p \otimes q)\left(A^{n} \otimes B^{m}\right)$.

Remark 3.3. Suppose that $E$ is an $A$-module satisfying the equivalent conditions of Theorem 3.1, and that $\varphi: A \rightarrow B$ is a non-degenerate $*$-homomorphism. Then we can write $E=p A^{n}$ for some $p \in \operatorname{Mult}(A)$. Since $\varphi$ is nondegenerate, it extends to a homomorphism $\tilde{\varphi}: \operatorname{Mult}(A) \rightarrow$ $\operatorname{Mult}(B)$, and we have $E \otimes_{\varphi} B \cong \tilde{\varphi}(p) B^{n}$. Hence $E \otimes_{\varphi} B$ also satisfies the equivalent conditions in Theorem 3.1.

The finite set $F$, and hence the unitisation $A_{b}$ of $A$, appearing in Theorem 3.1 is not canonical. The set $F$ depends on the choice of finite right basis $\left\{\xi_{j}\right\}$ for $\operatorname{Mult}(E)$ to which Kasparov's stabilisation theorem is applied in the second paragraph of the proof of Theorem 3.1. It is less obvious, but also true, that even if two choices of finite frame $\left\{\xi_{j}\right\},\left\{\xi_{j}^{\prime}\right\}$ yield the same unitisation $A_{b}$ of $A$, the enveloping projective modules $p A_{b}^{n}$ and $p^{\prime} A_{b}^{n}$ obtained from these frames may not be isomorphic, as we now demonstrate.

Example 3.4. We let $X:=\mathbb{C}$. Let $A=C_{0}(X)$, and let $E=C_{0}(X)$ the trivial module over $C_{0}(X)$ with the obvious inner product and multiplication action. Fix any countable locally finite open cover of $X$, fix a partition of unity $\left(\varphi_{n}\right)_{n}$ with respect to this cover, and set $e_{n}=\sqrt{\varphi_{n}}$ for each $n$. Then $\left\{e_{n}\right\}$ is a frame for $E$.

The module $\operatorname{Mult}(E)=\operatorname{Mult}(A)$ is a module over $\operatorname{Mult}(A)=C(\beta X)$, where $\beta X$ denotes the Stone-Čech compactification of $X$. Let us consider two choices of finite frame for this module. The first choice of frame has a single element

$$
x_{1}=1_{\operatorname{Mult}(A)} .
$$

The construction in the proof of Theorem 3.1 applied to this frame yields $A_{b}=C(\mathbb{C} \cup\{\infty\}) \cong$ $C\left(S^{2}\right), n=1, p=1_{A_{b}}$ and hence $p A_{b}^{n}=A_{b}$, the trivial module over $A_{b}$.

Now identify $\operatorname{Mult}(E)$ with $\left(\begin{array}{ll}1 & 0 \\ 0 & 0\end{array}\right)\left(\begin{array}{l}\operatorname{Mult}(A) \\ \operatorname{Mult}(A)\end{array}\right)$, and consider the elements $y_{i} \in \operatorname{Mult}(E)$ given by continuous extension of the functions

$$
y_{1}=\left(\begin{array}{c}
\frac{1}{\sqrt{1+|z|^{2}}} \\
0
\end{array}\right) \quad \text { and } \quad y_{2}=\left(\begin{array}{c}
\frac{\bar{z}}{\sqrt{1+|z|^{2}}} \\
0
\end{array}\right)
$$

to $\beta X$. Then $\left(y_{2} \mid y_{2}\right)_{\text {Mult }(A)}$ is identically 1 on the boundary $\beta X \backslash X$, and the other inner products $\left(y_{i} \mid y_{j}\right)_{\mathrm{Mult}(A)}$ are identically zero on the boundary. So all the $\left(y_{i} \mid y_{j}\right)_{\mathrm{Mult}(A)}$ take values in $C(X \cup\{\infty\})$, and hence the construction in the proof of Theorem 3.1 applied to this frame again yields $A_{b}=C(\mathbb{C} \cup\{\infty\}) \cong C\left(S^{2}\right)$. Now $n=2$, and calculation shows that

$$
p=\frac{1}{1+|z|^{2}}\left(\begin{array}{cc}
1 & \bar{z} \\
z & |z|^{2}
\end{array}\right) .
$$

Define $w \in M_{2}(\operatorname{Mult}(A))$ by continuous extension of the function

$$
w(z)=\left(\begin{array}{cc}
\frac{1}{\sqrt{1+|z|^{2}}} & 0 \\
\frac{z}{\sqrt{1+|z|^{2}}} & 0
\end{array}\right)
$$

to $\beta X$. We have

$$
w w^{*}=\frac{1}{1+|z|^{2}}\left(\begin{array}{cc}
1 & \bar{z} \\
z & |z|^{2}
\end{array}\right)=p \quad \text { and } \quad w^{*} w=\left(\begin{array}{cc}
1 & 0 \\
0 & 0
\end{array}\right) .
$$


So this $w$ defines an isomorphism of modules $p A^{2} \cong A$ over $A=C_{0}(X)$, but this does not extend to an isomorphism of modules over $A_{b}$ since $w$ is not well-defined on $S^{2}$. In particular, $p A_{b}^{2}$ is the module of sections of the Hopf line bundle over $S^{2} \cong \mathbb{C} \cup\{\infty\}$. This is certainly not trivial, and so not isomorphic to the trivial extension obtained for the first choice of frame above.

One important situation to which Theorem 3.1 applies is suspensions. Given a bimodule $E$ over an algebra $A$ we can define the suspended module $\mathcal{S} E$ over the suspension $\mathcal{S} A$ by

$$
\mathcal{S} E=C_{0}(\mathbb{R}) \otimes E, \quad\left(f_{1} \otimes a_{1}\right)(g \otimes e)\left(f_{2} \otimes a_{2}\right)=f_{1} g f_{2} \otimes a_{1} e a_{2}
$$

and with the obvious inner product.

Corollary 3.5. Let $A$ be a unital $C^{*}$-algebra and $E$ a finitely generated Hilbert module over $A$.

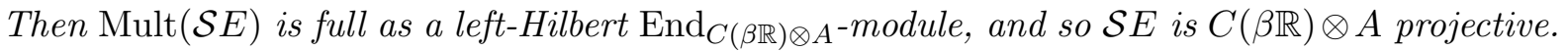
In fact $\operatorname{Mult}(\mathcal{S} E)$ is $A^{\sim}$ projective where $A^{\sim}$ is the minimal unitisation.

Proof. Given any frame $\left\{\xi_{j}\right\}$ for $E$, one checks that $\left\{1 \otimes \xi_{j}\right\}$ is a frame for $C(\beta \mathbb{R}) \otimes E$. So the result follows from Theorem 3.1.

The fullness hypothesis in Theorem 3.1 is quite restrictive. In particular, the multiplier module of a Hilbert module $E_{B}$ need not be full as a left $\operatorname{End}_{A}(E)$-module. For example, if $B$ is unital then $\operatorname{Mult}(E)=E$ [4], and so if $\operatorname{End}_{A}^{0}(E)$ is non-unital then $\operatorname{Mult}(E)$ is not full as a left $\operatorname{End}_{A}(E)$-module.

Example 3.6. Let $\mathcal{H}:=\ell^{2}(\mathbb{N})$ regarded as a $\mathcal{K}(\mathcal{H})-\mathbb{C}$-imprimitivity bimodule, and let $\overline{\mathcal{H}}$ denote the conjugate $\mathbb{C}-\mathcal{K}(\mathcal{H})$-equivalence. Let $A=\mathcal{K}(\mathcal{H}) \oplus \mathbb{C}$. Let $E:=\mathcal{H} \oplus \overline{\mathcal{H}}$ and define an $A$-bimodule structure on $E$ by

$$
\left(T_{1}, \lambda_{1}\right)\left(\xi_{1}, \overline{\xi_{2}}\right)\left(T_{2}, \lambda_{2}\right)=\left(T_{1} \xi_{1} \lambda_{2}, \lambda_{1} \overline{\xi_{2}} T_{2}\right)=\left(T_{1} \xi_{1} \lambda_{2}, \overline{T_{2}^{*} \xi_{2} \overline{\lambda_{1}}}\right)
$$

for all $T_{j} \in \mathcal{K}(\mathcal{H}), \lambda_{j} \in \mathbb{C}, \xi_{j} \in \mathcal{H}$. Define a right inner-product on $E$ by

$$
\left(\left(\xi_{1}, \overline{\xi_{2}}\right) \mid\left(\eta_{1}, \overline{\eta_{2}}\right)\right)_{\mathcal{K}(\mathcal{H}) \oplus \mathbb{C}}=\left(\left\langle\xi_{1}, \eta_{1}\right\rangle, \Theta_{\xi_{2}, \eta_{2}}\right) .
$$

Since $E$ decomposes as a direct sum of imprimitivity bimodules each of which has a unital algebra acting on one side or the other, the remark on pages 295 and 296 of [4] shows that $E$ is equal to its multiplier module $\operatorname{Mult}(E)$. The linking algebra is

$$
\left(\begin{array}{cc}
A & \mathcal{H} \oplus \overline{\mathcal{H}} \\
\overline{\mathcal{H}} \oplus \mathcal{H} & A
\end{array}\right)
$$

with multiplier algebra

$$
\left(\begin{array}{cc}
\mathcal{B}(\mathcal{H}) \oplus \mathbb{C} & \mathcal{H} \oplus \overline{\mathcal{H}} \\
\overline{\mathcal{H}} \oplus \mathcal{H} & \mathcal{B}(\mathcal{H}) \oplus \mathbb{C}
\end{array}\right)
$$

Hence $\operatorname{Mult}(E)=E=\mathcal{H} \oplus \overline{\mathcal{H}}$ is not full as a left $\operatorname{End}_{A}(E)$-module.

\section{Application to vector bundles}

Recall that for us a vector bundle is always a locally trivial, finite-rank, complex vector bundle equipped with a continuous family of inner products.

Theorem 4.1. Let $X$ be a second-countable locally compact Hausdorff space of finite topological dimension. 
a) Suppose that $V \rightarrow X$ is a vector bundle of rank $n$. Then there exists a vector bundle $\tilde{V}$ of rank $n$ over the Stone- $\check{C}$ ech compactification $\beta X$ such that $\left.V \cong \tilde{V}\right|_{X}$.

b) There is a metrisable compactification $X^{c}$ and a vector bundle $V^{c} \rightarrow X^{c}$ such that $V=$ $\left.V^{c}\right|_{X}$

Proof. (a) Let $E=\Gamma_{0}(X, V)$, and let $A=C_{0}(X)$. By Theorems 2.2 and 3.1, it suffices to show that $\operatorname{Mult}(E)$ is full as a left $\operatorname{End}_{A}(E)$-module. Using $\operatorname{Mult}(E) \cong \operatorname{Hom}_{A}(A, E)$, we see that every continuous bounded section of $V$ defines an element of $\operatorname{Mult}(E)$.

Fix a countable open cover $\mathcal{U}$ of $X$ by sets on which $V$ is trivial. Since $X$ has finite topological dimension, say $\operatorname{dim}(X)=d$, we can assume, by refining if necessary, that there is a partition

$\mathcal{U}=\bigsqcup_{i=0}^{d} \mathcal{U}_{i}$ such that distinct elements $U_{1}, U_{2}$ of any given $\mathcal{U}_{i}$ are disjoint. Fix a partition of unity $\left\{h_{U}: U \in \mathcal{U}\right\}$ and choose linearly independent sections $\left\{f_{U, j}: U \in \mathcal{U}, j=1, \ldots, n\right\}$ of $\left.V\right|_{U}$ such that

$$
\sum_{j=1}^{n} \operatorname{End}_{A}(E)\left(f_{U, j}(x) \mid f_{U, j}(x)\right)=\sum_{j=1}^{n} \Theta_{f_{U, j}(x), f_{U, j}(x)}=h_{U}(x) \operatorname{Id}_{V}
$$

for all $x, U$. For each $0 \leq i \leq d$ and $1 \leq j \leq n$, the pointwise sum $F_{i, j}:=\sum_{U \in \mathcal{U}_{i}} f_{U, j}$ is a bounded section of $V$ and so belongs to $\operatorname{Mult}(E)$. We then have

$$
\operatorname{Id}_{V}=\sum_{i=1}^{d} \sum_{j=1}^{n} \operatorname{End}_{A}(E)\left(F_{i, j} \mid F_{i, j}\right)
$$

so $\operatorname{Mult}(E)$ is full as a left $\operatorname{End}_{A}(E)$-module. A similar $\operatorname{argument}$ shows that $\operatorname{Mult}(E)$ is a full right $C(\beta X)$-module.

For (b), note that since $X$ is second-countable, $C_{0}(X)$ is separable. Thus by part (1) of Theorem 3.1 we see that $E$ is $A_{b}$-finite projective for a separable unitization $A_{b}$ of $C_{0}(X)$. So $A_{b} \cong C\left(X^{c}\right)$ for some second-countable, and hence metrisable, compactification of $X$. The module of sections of the restriction of $\tilde{V}$ to $X^{c}$ is then a finite projective module over $C\left(X^{c}\right)$.

Corollary 4.2. Let $A$ be nonunital, separable and commutative, so $A \cong C_{0}(X)$ and suppose that $X$ is of finite topological dimension. Let $E$ be a right $C^{*}-A$-module. Then the conditions (1)-(4) of Theorem 3.1 are equivalent to

5. There is a vector bundle $V \rightarrow X$ such that $E \cong \Gamma_{0}(X, V)$.

Proof. Suppose $E$ satisfies (5). By Theorem 4.1, the vector bundle $V$ extends to a bundle $\tilde{V}$ on the Stone-Cech compactification. By Theorem 2.2, $E=\Gamma_{0}(X, \tilde{V} \mid X) \cong p\left(C(\beta X)^{N}\right)$ for some $N$ and some $p \in M_{N}\left(C(\beta X)\right.$ ), and so $E$ satisfies (1). Conversely, if $E$ is $A_{b}$-finite projective for some unitisation $A_{b}$, then Theorem 2.2 implies that there is a vector bundle $\tilde{V}$ over the spectrum of $A_{b}$ such that $E \cong \Gamma_{0}\left(X,\left.\tilde{V}\right|_{X}\right)$. This completes the proof.

Corollary 4.3. Suppose that $X$ is a locally compact Hausdorff space with finite topological dimension. Then a right $C^{*}-C_{0}(X)$-module $E$ is $C\left(X^{c}\right)$ finite projective for some compactification $X^{c}$ of $X$ if and only if $E \cong \Gamma_{0}(X, V)$ for some vector bundle $V \rightarrow X$.

\section{Multiplier modules of bi-Hilbertian bimodules}

In this section, we investigate the structure of the multiplier module of a bi-Hilbertian bimodule in the sense of Kajiwara-Pinzari-Watatani (see Definition 5.1 below). We show that if $E$ 
has finite Watatani index then its multiplier module can always be made into a bi-Hilbertian bimodule with finite numerical index in its own right.

We then show that the passage of finite Watatani index from $E$ to $\operatorname{Mult}(E)$ is much less common; it is equivalent, for example, to fullness of $\operatorname{Mult}(E)$ as a left-Hilbert $\operatorname{End}_{A}(E)$-module. In particular, finite Watatani index does not help to weaken the fullness hypotheses of Theorem 3.1.

Definition 5.1 ([6, Definition 2.3]). Let $A$ be a $\sigma$-unital $C^{*}$-algebra. A bi-Hilbertian A-bimodule is a countably generated full right Hilbert $C^{*}-A$-module with inner product $(\cdot \mid \cdot)_{A}$ which is also a countably generated full left Hilbert $C^{*}-A$-module with inner product ${ }_{A}(\cdot \mid \cdot)$ such that the left action of $A$ is adjointable with respect to $(\cdot \mid \cdot)_{A}$ and the right action of $A$ is adjointable with respect to $A(\cdot \mid \cdot)$.

Since a bi-Hilbertian bimodule is complete in the norms coming from both the left and the right inner products, these two norms are equivalent.

Let $E$ be a bi-Hilbertian $A$-bimodule. We recall from [6, Definition 2.8] the definition of the right numerical index of $E$. We say that $E$ has finite right numerical index if there exists $\lambda>0$ such that

$$
\left\|\sum_{i} A\left(f_{i} \mid f_{i}\right)\right\| \leq \lambda\left\|\sum_{i} \Theta_{f_{i}, f_{i}}\right\| \quad \text { for all } n \text { and all } f_{1}, \ldots, f_{n} \in E .
$$

The right numerical index of $E$ is the infimum of the numbers $\lambda$ satisfying the above inequality.

Let $E$ be a bi-Hilbertian $A$-bimodule, countably generated as a right module and with finite right numerical index. By [6, Corollaries 2.24 and 2.28], the left action of $A$ on $E$ is by compact endomorphisms with respect to $(\cdot \mid \cdot)_{A}$ if and only if, for every frame $\left\{e_{j}\right\}$ for $\left(E,(\cdot \mid \cdot)_{A}\right)$, the series

$$
\sum_{j \geq 1} A\left(e_{j} \mid e_{j}\right)
$$

converges strictly in $\operatorname{Mult}(A)$. In this case we denote the strict limit by $r$-Ind $(E)$. We note that $r$ - $\operatorname{Ind}(E)$ is independent of the frame $\left\{e_{j}\right\}$, and is called the right Watatani index of $A$. This $r$ - $\operatorname{Ind}(E)$ is a positive central element of $\operatorname{Mult}(A)$, and is invertible if and only if the left action of $A$ is implemented by an injective homomorphism $A \rightarrow \operatorname{End}_{A}^{0}(E)$.

As in [6], we simply say that $E$ has finite right Watatani index if it has finite right numerical index and the left action is by compacts.

Theorem 5.2. Let $A$ be a nonunital $\sigma$-unital $C^{*}$-algebra and let $E$ be a bi-Hilbertian A-bimodule, countably generated as a right module and with injective left action. Suppose that $E$ has finite right Watatani index. Then ${ }_{A}(\cdot \mid \cdot)$ extends to a strictly continuous $\operatorname{Mult}(A)$-valued inner product on $\operatorname{Mult}(E)$, with respect to which $\operatorname{Mult}(E)$ is a bi-Hilbertian $\operatorname{Mult}(A)$-bimodule with finite right numerical index.

Proof. When the bi-Hilbertian $A$-bimodule $E$ has finite right Watatani index, [6, Corollary 2.11] shows that there is a positive $A$-bilinear norm continuous map $\Phi: \operatorname{End}_{A}^{0}(E) \rightarrow A$ such that

$$
{ }_{A}(e \mid f)=\Phi\left(\Theta_{e, f}\right), \quad e, f \in E .
$$

Proposition 2.27 of [6] implies that $\Phi$ extends to a bounded strictly continuous positive $A$-bilinear $\operatorname{map} \bar{\Phi}: \operatorname{End}_{A}(E) \rightarrow \operatorname{Mult}(A)$ with $\|\bar{\Phi}\| \leq \| r$-Ind $(E) \|$.

Now let $\operatorname{Mult}(E)$ be the multiplier module of $E$. The inclusion $A \hookrightarrow \operatorname{End}_{A}^{0}(E) \subseteq \mathcal{L}(E)$ implementing the left action extends to a homomorphism $\operatorname{Mult}(A) \rightarrow \operatorname{End}_{A}(E) \subseteq \operatorname{Mult}(\mathcal{L}(E))$,

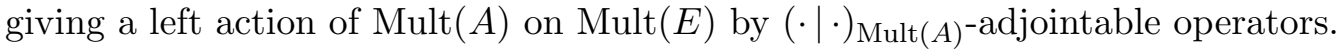


We saw at (2.1) that for $e, f \in \operatorname{Mult}(E)$ the operator $\Theta_{e, f} \in \operatorname{End}_{\operatorname{Mult}(A)}^{0}(\operatorname{Mult}(E))$ restricts to an adjointable operator on $E$, so we can define $\operatorname{Mult}(A)(\cdot \mid \cdot): \operatorname{Mult}(E) \times \operatorname{Mult}(E) \rightarrow \operatorname{Mult}(A)$ by

$$
\operatorname{Mult}(A)(e \mid f):=\bar{\Phi}\left(\Theta_{e, f}\right) .
$$

By definition of $\Phi$, this strictly continuous form extends ${ }_{A}(\cdot \mid \cdot)$.

We claim that this is a left-Mult $(A)$-linear $\operatorname{Mult}(A)$-valued inner-product on $\operatorname{Mult}(E)$. To see this, observe that sesquilinearity of $(e, f) \mapsto \Theta_{e, f}$, linearity of restriction and linearity of $\bar{\Phi}$ show that $\operatorname{Mult}(A)(\cdot \mid \cdot)$ is sesquilinear. For $a \in \operatorname{Mult}(A)$ and $e, f \in \operatorname{Mult}(E)$, we use the $A$-bilinearity of $\bar{\Phi}$ to see that

$$
a \cdot \operatorname{Mult}(A)(e \mid f)=a \bar{\Phi}\left(\left.\Theta_{e, f}\right|_{E}\right)=\bar{\Phi}\left(\left.a \Theta_{e, f}\right|_{E}\right)=\bar{\Phi}\left(\left.\Theta_{a \cdot e, f}\right|_{E}\right)={ }_{\operatorname{Mult}(A)}(a \cdot e \mid f),
$$

so $\operatorname{Mult}(A)(\cdot \mid \cdot)$ is left- $A$-linear. It is positive because $\bar{\Phi}$ is.

By [6, Proposition 2.27(1)], the maximal $\lambda^{\prime}>0$ such that $\lambda^{\prime}\left\|(e \mid e)_{A}\right\| \leq\|A(e \mid e)\|$ for all $e \in E$ satisfies

$$
\lambda^{\prime} T \leq \bar{\Phi}(T) \leq\|T\| r-\operatorname{Ind}(E) \quad \text { for all } 0 \leq T \in \operatorname{End}_{A}(E) .
$$

Hence $\operatorname{Mult}(A)(\cdot \mid \cdot)$ is positive definite. The same inequality combined with the norm equality $\left\|\Theta_{e, e}\right\|=\left\|(e \mid e)_{\operatorname{Mult}(A)}\right\|$ gives

$$
\lambda^{\prime}\left\|(e \mid e)_{\operatorname{Mult}(A)}\right\| \leq\left\|_{\operatorname{Mult}(A)}(e \mid e)\right\| \leq\|r-\operatorname{Ind}(E)\|\left\|(e \mid e)_{\operatorname{Mult}(A)}\right\|,
$$

and so the norms on $\operatorname{Mult}(E)$ coming from the left and right inner products are equivalent.

To see that $\operatorname{Mult}(E)$ has finite right numerical index, let $g_{1}, \ldots, g_{m} \in \operatorname{Mult}(E)$ be any finite set. Since $\bar{\Phi}$ is bounded we have

$$
\left\|\sum_{i \operatorname{Mult}(A)}\left(g_{i} \mid g_{i}\right)\right\|=\left\|\sum_{i} \bar{\Phi}\left(\Theta_{g_{i}, g_{i}}\right)\right\| \leq\|\bar{\Phi}\|\left\|\sum_{i} \Theta_{g_{i}, g_{i}}\right\| .
$$

In particular, given a frame $\left\{g_{j}: j \in \mathbb{N}\right\}$ for $\operatorname{Mult}(E)$ and any finite subset $I$ of $\mathbb{N}$, we have

$$
\left\|\sum_{i \in I} \operatorname{Mult}(A)\left(g_{i} \mid g_{i}\right)\right\| \leq\|\bar{\Phi}\| \text {. }
$$

So $\operatorname{Mult}(E)$ has finite right numerical index.

Our next theorem shows that while finite right numerical index passes easily from $E$ to $\operatorname{Mult}(E)$ as above, finite right Watatani index is another question.

Theorem 5.3. Let $A$ be a $\sigma$-unital non-unital $C^{*}$-algebra and let $E$ be a bi-Hilbertian $A$-bimodule, countably generated as a right module and with injective left action. Suppose that $E$ has finite right Watatani index. Then the following are equivalent:

1) $\operatorname{Mult}(E)$ is full as a left $\operatorname{Mult}(A)$-module;

2) $\operatorname{Id}_{\operatorname{Mult}(E)}$ is a compact endomorphism of $\operatorname{Mult}(E)_{\operatorname{Mult}(A)}$;

$3)$ the left action of $\operatorname{Mult}(A)$ on $\operatorname{Mult}(E)_{\operatorname{Mult}(A)}$ is by compact endomorphisms;

4) $r$-Ind $(\operatorname{Mult}(E)) \in \operatorname{Mult}(A)$ (that is, $\operatorname{Mult}(E)$ has finite right Watatani index); and

5) $E$ is $\operatorname{Mult}(A)$-finite projective as in Definition 2.3.

Proof. The left action of $\operatorname{Mult}(A)$ is injective. Hence [6, Corollaries 2.20 and 2.28] gives $(1) \Leftrightarrow(4)$. We have $(2) \Leftrightarrow(3)$ because $\operatorname{End}_{\operatorname{Mult}(A)}^{0}(\operatorname{Mult}(E))$ is an ideal of $\operatorname{End}_{\operatorname{Mult}(A)}(\operatorname{Mult}(E))$ and because the identity of $\operatorname{Mult}(A)$ acts as $\operatorname{Id}_{\operatorname{Mult}(E)}$. Similarly, $(5) \Rightarrow(3)$ since in this case all the endomorphisms are compact. The implication $(1) \Rightarrow(5)$ follows from the implication $(4) \Rightarrow(2)$ in Theorem 3.1. So it suffices to prove that $(3) \Leftrightarrow(4)$, which follows from [6, Theorem 2.22]. 
Remark 5.4. If the equivalent conditions (1)-(5) of Theorem 5.3 hold, then Theorem 3.1 shows that in fact $E$ is $A_{b}$-finite projective for some subalgebra of $\operatorname{Mult}(A)$ generated by $A$ and just finitely many additional elements.

Remark 5.5. By Theorem 5.2, if the equivalent conditions in Theorem 5.3 hold, then the left inner product on $E$ extends by strict continuity to a $\operatorname{Mult}(A)$-valued left inner product on $\operatorname{Mult}(E)$ under which $\operatorname{Mult}(E)$ itself becomes a bi-Hilbertian bimodule with invertible finite right Watatani index.

Example 5.6. We describe a concrete example where Theorem 5.2 applies but the equivalent conditions in Theorem 5.3 do not hold. Consider the non-unital $C^{*}$-algebra $A:=\mathcal{K}$, the compact operators on $\ell^{2}(\mathbb{N})$. Let $E$ be the external tensor product $E=\ell^{2} \otimes \mathcal{K}$, which is a Morita equivalence from $\mathcal{K} \cong \mathcal{K} \otimes \mathcal{K}$ to $\mathbb{C} \otimes \mathcal{K} \cong \mathcal{K}$, and hence an $A-A$-imprimitivity bimodule. So $E$ has finite right Watatani index $r-\operatorname{Ind}(E)=1_{M(\mathcal{K})}$.

It is routine to check that $\operatorname{Mult}(E) \cong \ell^{2} \otimes \mathcal{B}\left(\ell^{2}\right)$. Since the left action of $\operatorname{Mult}(A)=\mathcal{B}\left(\ell^{2}\right)$ on this module is not by compact operators, Theorem $5.3(2)$ fails, and therefore so do the other conditions. In particular, while $\operatorname{Mult}(E)$ has finite numerical index by Theorem 5.2 , it does not have finite right Watatani index.

Finite right Watatani index can nevertheless be a useful tool for deciding when we obtain finite projective modules over unitisations. For a commutative algebra $A$, we can equip a right inner product module $E$ over $A$ with a left action and inner product via the formulae

$$
a \cdot e:=e a, \quad \text { and } \quad{ }_{A}\left(e_{1} \mid e_{2}\right):=\left(e_{2} \mid e_{1}\right)_{A}, \quad \text { for all } e, e_{1}, e_{2} \in E, a \in A .
$$

So we are in the bi-Hilbertian setting. For each character $\phi \in \widehat{A}$, the (completion of the) quotient $\mathcal{H}_{\phi}:=E /(E \cdot \operatorname{ker} \phi)$ is a Hilbert space with inner product $\left\langle e_{1}, e_{2}\right\rangle=\phi\left(\left(e_{1} \mid e_{2}\right)_{A}\right)$.

Corollary 5.7. Let $X$ be a second-countable locally compact Hausdorff space of finite topological dimension, and let $A=C_{0}(X)$. Let $E$ be a full right Hilbert $A$-module, regarded as a bi-Hilbertian bimodule as in (5.1). The following are equivalent:

1) The map $\phi \mapsto \operatorname{dim} \mathcal{H}_{\phi}$ is a continuous bounded function from $X$ to $[0, \infty)$;

2) $E$ is isomorphic to $\Gamma_{0}(X, V)$ for some (finite rank) vector bundle $V \rightarrow X$;

3) E has finite right Watatani index.

Proof. We first prove that (1) implies (2). For $e \in E$, define a section $S_{e}: X \rightarrow \bigcup_{\phi \in X} \mathcal{H}_{\phi}=$ $E /(E \cdot \operatorname{ker} \phi)$ by $S_{e}(\phi)=e+E \cdot \operatorname{ker} \phi$. This set of sections is a vector space and is fibrewise dense (in fact, fibrewise surjective), and so by [5, Theorem II.13.18] there is a unique topology on $V:=\bigcup_{\phi} \mathcal{H}_{\phi}$ under which these sections are continuous.

For $a \in C_{0}(X)$ and $e \in E$, we see that $S_{e \cdot a}(\phi)=a(\phi) S_{e}(\phi)$ for all $\phi \in \hat{A}$. So [5, Corollary II.14.7] shows that the $S_{e}$ are uniformly-on-compacta dense in $\Gamma_{0}(X, V)$. A simple argument using completeness of $E$ then shows that $e \mapsto S_{e}$ is a surjection of $E$ onto $\Gamma_{0}(X, V)$. By definition, this surjection carries the right action and inner product on $E$ to those on $\Gamma_{0}(X, V)$. So $e \mapsto S_{e}$ is an isomorphism $E \cong \Gamma_{0}(X, V)$.

We must check that $V$ is locally trivial. Remark II.13.9 of [5] states that finite-rank continuous bundles of Banach spaces of constant dimension are locally trivial; we provide a proof in our setting for completeness (see also [3, Remarque, p. 231]).

Fix $\phi \in X$, and choose an orthonormal basis $\left\{e_{1}, \ldots, e_{n}\right\}$ for $\mathcal{H}_{\phi}$. Fix elements $\xi_{i} \in E$ such that $\xi_{i}+E \cdot \operatorname{ker} \phi=e_{i}$. By scaling by an appropriate element of $C_{0}(X)$ we can assume that there is a neighbourhood $U$ of $\phi$ such that for $\psi \in U$, the element $e_{i}^{\psi}:=\xi_{i}+E \cdot \operatorname{ker} \psi$ satisfies $\left\|e_{i}^{\psi}\right\|=1$. Since $\psi \mapsto \operatorname{dim} \mathcal{H}_{\psi}$ is continuous, and since $\left(\xi_{i} \mid \xi_{j}\right)$ is continuous for all $i$, $j$, by 
shrinking $U$ if necessary we can assume that $\operatorname{dim} \mathcal{H}_{\psi}=n$ for all $\psi \in U$ and that $\left|\left\langle e_{i}^{\psi}, e_{j}^{\psi}\right\rangle\right|<\frac{1}{2 n^{2}}$ for $i \neq j$ and $\psi \in U$. Now if $\psi \in U$ and $\sum_{i} \alpha_{i} e_{i}^{\psi}=0$, then

$$
0=\left|\left\langle\sum_{i} \alpha_{i} e_{i}^{\psi}, \sum_{j} \alpha_{j} e_{j}^{\psi}\right\rangle\right| \geq \sum_{i}\left|\alpha_{i}\right|^{2}\left\|e_{i}^{\psi}\right\|^{2}-\frac{\left(n^{2}-n\right) \max _{i}\left|\alpha_{i}\right|^{2}}{2 n^{2}} \geq \max _{i}\left|\alpha_{i}\right|^{2} / 2 .
$$

So $V$ admits a continuous choice of basis on $U$, so is locally trivial.

To see that (2) implies (3), suppose that $E$ has the form $\Gamma_{0}(X, V)$. Choose a locally finite cover of $X$ by sets $U_{i}$ on which on which $V$ is trivial, say $\left.V\right|_{U_{i}} \cong U_{i} \times \mathbb{C}^{n_{i}}$. Since $V$ is finite rank, $\sup _{i} n_{i}<\infty$. Let $e_{1}, \ldots, e_{j}$ denote the bounded sections of $\left.V\right|_{U_{i}}$ given by the standard basis of $\mathbb{C}^{n_{i}}$.

Choose a partition of unity $\left(h_{i}\right)$ subordinate to the $U_{i}$, and put $f_{i, j}=\sqrt{h_{i}} e_{j}$ for $1 \leq j \leq n_{i}$ and $i \in \mathbb{N}$. Then just as in Theorem 4.1 we see that

$$
\sum_{i, j} \Theta_{f_{i, j}, f_{i, j}}=\sum_{i} h_{i} \operatorname{Id}_{\left.V\right|_{U_{i}}}=\mathrm{Id}_{V}
$$

Using the module structure defined in (5.1), we see that the left inner product of two sections $e=\sum_{j} c_{j} e_{j}, f=\sum_{k} d_{k} e_{k}$ supported in $U_{i}$ is given by

$$
\begin{aligned}
{ }_{A}(e \mid f) & =\sum_{j, k} c_{j} \overline{d_{k}}\left(e_{k} \mid e_{j}\right)_{A}=\sum_{j} c_{j} \overline{d_{j}}=\sum_{j}\left(e_{j} \mid e\right)_{A}\left(f \mid e_{j}\right)_{A}=\sum_{j}\left(e_{j} \mid \Theta_{e, f} e_{j}\right)_{A} \\
& =\operatorname{Trace}\left(\Theta_{e, f}\right) .
\end{aligned}
$$

Hence for any finite set $\left\{e_{k}: k \leq K\right\} \subseteq E$, we have

$$
\left\|\sum_{k} A\left(e_{k} \mid e_{k}\right)\right\| \leq\left(\sup _{i} n_{i}\right)\left\|\sum_{k} \Theta_{e_{k}, e_{k}}\right\|
$$

and so $E$ has finite numerical index.

This also shows that $\sum_{j} A\left(f_{i, j} \mid f_{i, j}\right)=h_{i} n_{i}$ on the open set $U_{i}$, and so $\left(\sum_{i, j} A\left(f_{i, j} \mid f_{i, j}\right)\right) a$ converges to $n_{i} a$ for $a \in C_{c}\left(U_{i}\right)$. So $\sum_{i, j} A\left(f_{i, j} \mid f_{i, j}\right)$ converges strictly. That is, $E$ has finite right Watatani index.

Finally, for (3) implies (1), suppose that $E$ has finite right Watatani index. By definition of the bi-Hilbertian structure (5.1), the right Watatani index of $E$ is the function $\phi \mapsto \operatorname{dim} \mathcal{H}_{\phi}$. By definition of finite Watatani index, we deduce that $\left(\phi \mapsto \operatorname{dim} \mathcal{H}_{\phi}\right) \in \operatorname{Mult}\left(C_{0}(X)\right) \cong C_{b}(X)$, giving (1).

\section{Acknowledgements}

This research was supported by Australian Research Council grant DP150101595. It was motivated by questions arising in projects with our collaborators Francesca Arici, Magnus Goffeng, Bram Mesland and Dave Robertson, and we thank them for all that we have learned from them. We are very grateful to the anonymous referee who read the manuscript very closely and made numerous very helpful suggestions that have significantly strengthened our results and streamlined our proofs. Thanks, whoever you are.

\section{References}

[1] Arambašić L., Bakić D., Frames and outer frames for Hilbert $C^{*}$-modules, Linear Multilinear Algebra 65 (2017), 381-431, arXiv:1507.04101. 
[2] Blecher D.P., Le Merdy C., Operator algebras and their modules - an operator space approach, London Mathematical Society Monographs, Oxford Science Publications, Vol. 30, The Clarendon Press, Oxford University Press, Oxford, 2004.

[3] Dixmier J., Douady A., Champs continus d'espaces hilbertiens et de $C^{*}$-algèbres, Bull. Soc. Math. France 91 (1963), 227-284.

[4] Echterhoff S., Raeburn I., Multipliers of imprimitivity bimodules and Morita equivalence of crossed products, Math. Scand. 76 (1995), 289-309.

[5] Fell J.M.G., Doran R.S., Representations of *-algebras, locally compact groups, and Banach *-algebraic bundles, Vol. 1, Basic representation theory of groups and algebras, Pure and Applied Mathematics, Vol. 125, Academic Press, Inc., Boston, MA, 1988.

[6] Kajiwara T., Pinzari C., Watatani Y., Jones index theory for Hilbert $C^{*}$-bimodules and its equivalence with conjugation theory, J. Funct. Anal. 215 (2004), 1-49, arXiv:math.OA/0301259.

[7] Raeburn I., Thompson S.J., Countably generated Hilbert modules, the Kasparov stabilisation theorem, and frames with Hilbert modules, Proc. Amer. Math. Soc. 131 (2003), 1557-1564.

[8] Raeburn I., Williams D.P., Morita equivalence and continuous-trace $C^{*}$-algebras, Mathematical Surveys and Monographs, Vol. 60, Amer. Math. Soc., Providence, RI, 1998.

[9] Rennie A., Smoothness and locality for nonunital spectral triples, K-Theory 28 (2003), 127-165.

[10] Rennie A., Robertson D., Sims A., The extension class and KMS states for Cuntz-Pimsner algebras of some bi-Hilbertian bimodules, J. Topol. Anal. 9 (2017), 297-327, arXiv:1501.05363.

[11] Swan R.G., Vector bundles and projective modules, Trans. Amer. Math. Soc. 105 (1962), 264-277. 